\title{
Protective role of statins in COVID 19 patients: importance of pharmacokinetic characteristics rather than intensity of action
}

\author{
Rosario Rossi ${ }^{1}\left[\right.$ [ $\cdot$ Marisa Talarico ${ }^{1} \cdot$ Francesca Coppi $^{1} \cdot$ Giuseppe Boriani $^{1}$
}

Received: 25 June 2020 / Accepted: 12 September 2020 / Published online: 3 October 2020

(c) Società Italiana di Medicina Interna (SIMI) 2020

\section{Dear Editor,}

Statins represent a family of drugs that are potentially able to defend COVID-19 patients against uncontrolled systemic inflammatory response produced by the virus Sars-Cov- 2 . Therefore, some physicians proposed and used anti-inflammatory agents in the treatment regimen of patients with COVID-19 [1]. Statins are well known for their anti-inflammatory effects [2], and some hospitals included them in the COVID-19 treatment protocol [3]. In addition, studies in vitro verified that "there is evidence suggesting that statins exert anti-viral activity and may block the infectivity of enveloped viruses" [4]. In other words, statins could be efficient SARS-CoV-2 inhibitors of the main protease, a key coronavirus enzyme, which is a potential drug target [4].

Considering the above premises, we hypothesized that patients taking statins were better protected against mortality risk than those who do not take statins. We verified this hypothesis in a population of 71 consecutive patients with a pre-existing chronic cardiovascular disease, who become ill from COVID-19 between February 29, 2020, and May 20, 2020. The follow-up ended on June 15, 2020. The only endpoint of the study was all-cause mortality.

Continuous variables were expressed as mean \pm one SD or median (range) values; and categorical data as percentages. All dichotomous variables were compared utilizing the $\chi 2$ test; and continuous parameters using analysis of variance (ANOVA) or Mann-Whitney $U$ test, as appropriate. Survival probabilities were estimated with the Kaplan-Meier method and survival curves plotted and compared between groups using the log-rank test. $P<0.05$ was considered statistically significant.

Rosario Rossi

rosario.rossi@unimore.it

1 Cardiology Unit, Policlinico Di Modena Hospital, University of Modena and Reggio Emilia, Via del Pozzo 71, 41124 Modena, Italy
The baseline characteristics of the studied population were illustrated in Table 1. Groups were created according to the assumption, or not, of statins.

The mortality rate of patients taking statins resulted in $21.4 \%$ ( 9 of 42 patients died during the observation period in this group), while in the group of patients without statins it was $34.5 \%$ (10 of 29 patients); $p<0.05$. The comparison of the survival curves showed a statistically not significant difference (Fig. 1a). In other words, there is a trend towards a reduction in mortality risk, but the effect of statins seems substantially not significant.

As a further analysis, to investigate whether the intensity of action of statins could influence the risk of mortality, we divided our 42 patients taking statins into two subgroups, based on the drug's intensity of action, according to the ACC/AHA Classification [5]. The results were that 18 patients of 42 assumed a high-intensity statin ( 8 patients of these rosuvastatin $20 \mathrm{mg} / \mathrm{die} ; 8$ patients atorvastatin $40 \mathrm{mg} / \mathrm{die}$; and 2 patients atorvastatin $80 \mathrm{mg} / \mathrm{die}$ ), and 24 patients/42 a low- or moderate-intensity statin (6 patients rosuvastatin $10 \mathrm{mg} / \mathrm{die} ; 2$ patients pravastatin $40 \mathrm{mg} / \mathrm{die}$; 2 patients atorvastatin $10 \mathrm{mg} / \mathrm{die} ; 4$ patients simvastatin $20 \mathrm{mg} / \mathrm{die}$; and 14 patients atorvastatin $20 \mathrm{mg} / \mathrm{die}$ ). In the subgroup of high-intensity statins, 4 patients of $18(22.2 \%)$ died, with respect to 6 patients of $24(25.0 \%)$ in the lowmoderate-intensity group. The comparison between the survival curves, in contrast to patients who did not take statins, showed no significant differences (Fig. 1b).

To demonstrate whether the pharmacokinetic characteristics of statins were able to determine cardiovascular protection, we divided our 42 patients taking statins into two subgroups, based on the solubility of the used statin. The result was that 16 patients of 42 (38.1\%) took a watersoluble (hydrophilic) statin (rosuvastatin in 14 patients and pravastatin in 2), while 26/42 (61.9\%) a lipid-soluble (lipophilic) statin (atorvastatin in 22 patients, and simvastatin in $4)$. The comparison between the survival curves, in contrast to patients who did not take statins, indicated a significant 
Table 1 Baseline characteristics of the study population

\begin{tabular}{|c|c|c|c|}
\hline Parameter & Statin YES & Statin NO & $p$ \\
\hline$n$ & $42(59.1 \%)$ & $29(40.9 \%)$ & \\
\hline Age, years, median (range) & $71(64-92)$ & $73(63-90)$ & 0.5 \\
\hline \multicolumn{4}{|l|}{ Motivation of statin administration } \\
\hline Post myocardial infarction & $47.6 \%(n=20)$ & & \\
\hline Post stroke & $23.8 \%(n=10)$ & & \\
\hline Prevention & $28.5 \%(n=12)$ & & \\
\hline \multicolumn{4}{|l|}{ Risk factors for cardiovascular diseases } \\
\hline Male gender & $57.1 \%(n=24)$ & $55.2 \%(n=16)$ & 0.5 \\
\hline Hypertension & $71.4 \%(n=30)$ & $68.9 \%(n=20)$ & 0.4 \\
\hline Type II Diabetes Mellitus & $76.2 \%(n=32)$ & $27.6 \%(n=8)$ & 0.001 \\
\hline Hypercolesterolemia & $95.2 \%(n=40)$ & $13.8 \%(n=4)$ & 0.0001 \\
\hline Obesity $\left(\mathrm{BMI}>30 \mathrm{~kg} / \mathrm{m}^{2}\right)$ & $40.5 \%(n=17)$ & $37.9 \%(n=11)$ & 0.3 \\
\hline \multicolumn{4}{|l|}{ Pre-existing chronic cardiovascular diseases } \\
\hline Coronary artery disease & $85.7 \%(n=36)$ & $13.8 \%(n=4)$ & 0.001 \\
\hline Cerebro-vascular disease & $35.7 \%(n=15)$ & $10.3 \%(n=3)$ & 0.03 \\
\hline Aortic or Mitral valvulopathy & $11.9 \%(n=5)$ & $10.3 \%(n=3)$ & 0.7 \\
\hline Chronic heart failure & $52.4 \%(n=22)$ & $51.7 \%(n=15)$ & 0.8 \\
\hline Hystory of pulmonary embolism & $7.1 \%(n=3)$ & $6.9 \%(n=2)$ & 0.6 \\
\hline Chronic obstructive pulmonary disease & $19.0 \%(n=8)$ & $20.7 \%(n=6)$ & 0.1 \\
\hline Chronic renal failure & $16.7 \%(n=7)$ & $17.2 \%(n=5)$ & 0.3 \\
\hline \multicolumn{4}{|l|}{ Chronically taken drugs } \\
\hline Aspirin & $76.2 \%(n=32)$ & $51.7 \%(n=15)$ & 0.001 \\
\hline P2Y12 Inhibitors & $14.3 \%(n=6)$ & $13.8 \%(n=4)$ & 0.2 \\
\hline Beta-blockers & $71.4 \%(n=30)$ & $72.4 \%(n=21)$ & 0.3 \\
\hline DOAC & $23.8 \%(n=10)$ & $24.1 \%(n=7)$ & 0.4 \\
\hline ACEIs & $42.8 \%(n=18)$ & $44.8 \%(n=13)$ & 0.09 \\
\hline $\mathrm{ARBs}$ & $30.9 \%(n=13)$ & $31.0 \%(n=9)$ & 0.8 \\
\hline Calcium-antagonists & $14.3 \%(n=6)$ & $13.8 \%(n=4)$ & 0.2 \\
\hline
\end{tabular}

ACEIs angiotensin converting-enzyme inhibitors; ARBS angiotensin II receptors blockers; $B M I$ body mass index; DOAC direct oral anticoagulants difference between groups. Particularly, the group of lipophilic statins demonstrated a significant reduction in mortality respect both patients who do not take statins, and patients who assumed hydrophilic statins (Fig. 1c). No differences were found regarding clinical characteristics and lipid profile between patients who assumed hydrophilic or lipophilic statins (Table 2).

SARS-CoV-2 contaminates multiple cell types in different organs, binding glycoprotein angiotensin-converting enzyme 2 (ACE2), the critical receptor mediating the virus entry [6]. Many studies demonstrated that yet the lungs are massively deranged, COVID-19 infection can extend to many organs, including the blood vessels, heart, gut, and kidneys [6, 7]. Therefore, there are some abundantly infiltrated tissues, as they are particularly rich in ACE-2, which constitute the real "deposits of infection", from where the virus spreads. These tissues are the brain, central nervous system [8] and the adipose tissue [9]. Moreover, adipose cell embolism amplifies both the inflammatory phenomena and the pro-thrombotic status [9]. The latter is a well-known prognostic factor and chronic oral anti-coagulation showed a reduction in mortality in patients with chronic cardiac disease [10].

Given the availability, low cost, and safety of statins there was an intense debate concerning statins repurposing as part of COVID-19 treatment. In a recent study, no significant differences in statin chronic assumption were found in COVID19 patients who developed a cardiac injury, even if this population showed an increased mortality risk in the brief period [11]. As a matter of facts, the promising result of chronic statins intake should be further investigated in randomized controlled trials [12] even if their use was recently related to a reduced mortality risk [13].

Moreover, the beneficial role of statins in this context can be explained either by their immunomodulatory action or by preventing cardiovascular damage [14].

Lipophilic statins have a large distribution volume, reaching all the body's tissues, provide their protective 


\section{Panel A}

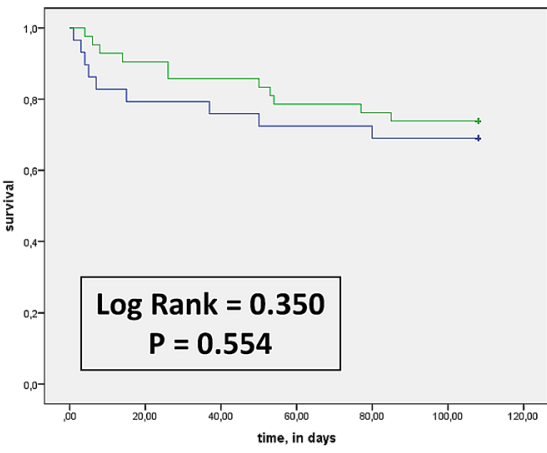

Green line $=$ YES statins Blue line $=$ NO statins
Panel B



Green line $=$ High-intensity statins

Yellow line $=$ Low- or Moderate-intensity statins

Blue line $=$ NO statins
Panel C

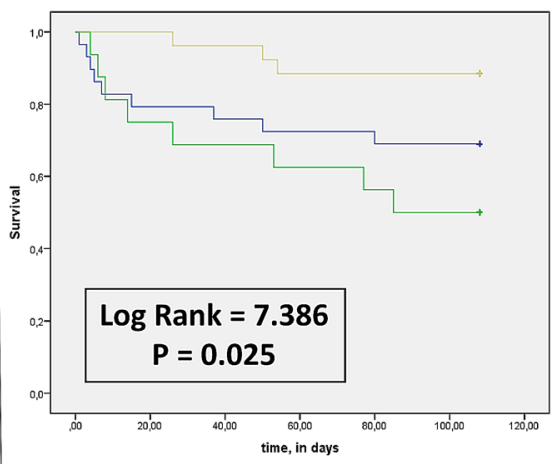

Green line $=$ hydrophilic statins Yellow line $=$ lipophilic statins Blue line $=$ NO statins
Fig. 1 Event-free survival in patients affected by COVID 19 in connection with taking statins. Unadjusted cumulative event rates for the primary end-point (all-cause mortality) was estimated using the Kaplan-Meier method, and matched between the groups using the log-rank test. In the panel a we appreciate the comparison between patients who taking statins respect to do not take statins. The comparison between the survival curves showed no significant differences.
In the panel $\mathbf{b}$ the contrast concerns patients who take high-intensity versus low- or moderate-intensity statins. The comparison between the survival curves, in contrast to patients who did not take statins, showed no significant differences. In the panel c confrontation pertains patients who take lipophilic versus hydrophilic statins. The comparison between the survival curves, in contrast to patients who do not take statins, showed a significant differences
Table 2 Comparisons between patients who take lipophilic and hydrophilic statins

\begin{tabular}{llll}
\hline Number of patients $=42$ & $\begin{array}{l}\text { High-intensity } \\
\text { statins } n=22\end{array}$ & $\begin{array}{l}\text { Low/moderate-intensity } \\
\text { statins } n=20\end{array}$ & $p$ value \\
\hline Clinical characteristics & & & \\
Age, years, median $($ range) & $70(64-92)$ & $72(64-92)$ & 0.7 \\
Obesity $\left(\mathrm{BMI}>30 \mathrm{~kg} / \mathrm{m}^{2}\right)$ & $40.9 \%(n=9)$ & $40.0 \%(n=8)$ & 0.9 \\
Male gender & $59.0 \%(n=13)$ & $55.0 \%(n=11)$ & 0.3 \\
Pre-existing chronic cardiovascular diseases & & & \\
Coronary artery disease & $86.3 \%(n=19)$ & $80.0 \%(n=16)$ & 0.1 \\
Cerebro-vascular disease & $36.3 \%(n=8)$ & $35.0 \%(n=7)$ & 0.7 \\
Aortic or mitral valvulopathy & $13.6 \%(n=3)$ & $10.0 \%(n=2)$ & 0.5 \\
Chronic heart failure & $59.0 \%(n=13)$ & $55.0 \%(n=10)$ & 0.3 \\
Hystory of pulmonary embolism & $0.90 \%(n=2)$ & $0.50 \%(n=1)$ & 0.8 \\
Chronic obstructive pulmonary disease & $18.1 \%(n=4)$ & $20.0 \%(n=4)$ & 0.6 \\
Chronic renal failure & $18.1 \%(n=4)$ & $15.0 \%(n=3)$ & 0.5 \\
Lipid profile (mg/dl) & & & \\
LDL & $89 \pm 13$ & $91 \pm 11$ & 0.8 \\
HDL & $57 \pm 11$ & $52 \pm 13$ & 0.5 \\
Triglycerides & $112 \pm 12$ & $110 \pm 15$ & 0.8 \\
Total Cholesterol & $167 \pm 16$ & $172 \pm 18$ & 0.5 \\
\hline
\end{tabular}


role against the virus. On the contrary, hydrophilic statins have some difficulties to permeate organs, including adipose tissue and tissues protected by functional barriers (i.e., blood-brain barrier). Therefore, even with the same lipidlowering efficacy, hydrophilic statins have less anti-inflammatory properties.

The current study has several limitations. First of all, this is an observational study, so it can provide associations but not causality. An additional limitation is the small sample size. Even if the current study provided a novel point of view, for the limitation discussed above, our findings need to further validate on a larger population and randomized trials.

In conclusion, statins have been able to significantly reduce the risk of mortality of the COVID 19 patient, provided that they manage to reach the sites where the virus creates damages and where it accumulates, that is, provided that lipophilic statins are used.

Author contributions RR and MT provided the acquisition, analysis, and interpretation of data and wrote the manuscript. FC and GB provided critical analysis of data and discussion and revised the manuscript critically for important intellectual content. All the authors gave a substantial contribution to the work and approved the version to be published.

Funding None.

\section{Compliance with ethical standards}

Conflict of interest The authors declare no conflict of interest.

Ethical standards This study was approved by the local Ethics Committee (Protocol Number AOU 0012597).

Statements on human and animal rights All procedures followed were in accordance with the Declaration of Helsinki.

Informed consent Informed consent was obtained from all individual participants included in the study.

\section{References}

1. Zhang W, Zhao Y, Zhang F et al (2020) The use of anti-inflammatory drugs in the treatment of people with severe coronavirus disease 2019 (COVID-19): the experience of immunologists from China. ClinImmunol. https://doi.org/10.1016/j.clim.2020.108393

2. Schönbeck U, Libby P (2004) Inflammation, immunity, and HMGCoA reductase inhibitors: statins as anti-inflammatory agents? Circulation 109:II-18-II-26

3. Massachusetts General Hospital COVID-19 Treatment Guidance Version 1.0 3/17/2020. Available at: https://medtube.net/infec tious-diseases/medical-documents/26086-covid19-treatmentguidelines-by-massachusetts-general-hospital. Accessed March 28, 2020.

4. Reiner Ž, Hatamipour M, Banach M et al (2020) Statins and the COVID-19 main protease: in silico evidence on direct interaction. Arch Med Sci 16(3):490-496

5. Stone NJ, Robinson J, Lichtenstein AH et al (2013) 2013 ACC AHA guideline on the treatment of blood cholesterol to reduce atherosclerotic cardiovascular risk in adult: a report of the american college of cardiology/american heart association task force on practice guidelines. J Am CollCardiol 63:2889-2934

6. Wadman M, Couzin-Frankel J, Kaiser J, Matacic C (2020) How does coronavirus kill? Clinicians trace a ferocious rampage through the body, from brain to toes. Science. https://doi. org/10.1126/science.abc3208

7. Perrella A, Trama U, Bernardi FF et al (2020) EditorialCOVID-19, more than a viral pneumonia. Eur Rev Med Pharmacol Sci 24(9):5183-5185

8. Mao L, Jin H, Wang M et al (2020) Neurologic manifestations of hospitalized patients with coronavirus disease 2019 in Wuhan, China. JAMANeurol 2020:e201127

9. Cinti S, Graciotti L, Giordano A, Valerio A, Nisoli E (2020) COVID-19 and fat embolism: a hypothesis to explain the severe clinical outcome in people with obesity. Int J Obes. https://doi. org/10.1038/s41366-020-0624-5

10. Rossi R, Coppi F, Talarico M, Boriani G (2020) Protective role of chronic tretment with direct oral anticoagulants in elderly patients affected by interstitial pneumonia in COVID-19 era. Europ J Intern Med. https://doi.org/10.1016/j.ejim.2020.06.006

11. Karbalai Saleh S, Oraii A, Soleimani A et al (2020) The association between cardiac injury and outcomes in hospitalized patients with COVID-19. Intern Emerg Med. https://doi.org/10.1007/ s11739-020-02466-1

12. Fajgenbaum DC, Rader DJ (2020) Teaching old drugs new tricks: statins for covID-19? Cell Metab 32(2):145-147

13. Zhang XJ, Qin JJ, Cheng X et al (2020) In-hospital use of statins is associated with a reduced risk of mortality among individuals with COVID-19. Cell Metab 32(2):176-187.e4

14. Castiglione V, Chiriacò M, Emdin M, Taddei S, Vergaro G (2020) Statin therapy in COVID-19 infection. Eur Heart J CardiovascPharmacother. https://doi.org/10.1016/j.cmet.2020.06.015

Publisher's Note Springer Nature remains neutral with regard to jurisdictional claims in published maps and institutional affiliations. 